\section{Maternal western diet causes inflammatory milk and TLR2/4-dependent neonatal toxicity}

\author{
Yang Du, ${ }^{1}$ Marie Yang, ${ }^{1}$ Syann Lee, ${ }^{2}$ \\ Cassie L. Behrendt, ${ }^{3}$ Lora V. Hooper, ${ }^{3,4}$ \\ Alan Saghatelian, ${ }^{5}$ and Yihong Wan ${ }^{1,6}$ \\ ${ }^{1}$ Department of Pharmacology, ${ }^{2}$ Department of Internal \\ Medicine, Division of Hypothalamic Research, ${ }^{3}$ Department \\ of Immunology, ${ }^{4}$ The Howard Hughes Medical Institute, \\ University of Texas Southwestern Medical Center, Dallas, \\ Texas 75390, USA; ${ }^{5}$ Department of Chemistry and Chemical \\ Biology, Harvard University, Cambridge, Massachusetts 02138, \\ USA
}

For all newborn mammals, mother's milk is the perfect nourishment, crucial for their postnatal development. Here we report that, unexpectedly, maternal western diet consumption in mice causes the production of toxic milk that contains excessive long chain and saturated fatty acids, which triggers ceramide accumulation and inflammation in the nursing neonates, manifested as alopecia. This neonatal toxicity requires Toll-like-receptors (TLR), but not gut microbiota, because TLR2/4 deletion or TLR4 inhibition confers resistance, whereas germ-free mice remain sensitive. These findings unravel maternal western diet-induced inflammatory milk secretion as a novel aspect of the metabolic syndrome at the maternal offspring interface.

Supplemental material is available for this article.

Received February 29, 2012; revised version accepted May 11, 2012.

Throughout history, mother's milk has been regarded as the perfect food. Rich, nutritious, and protective, it is the drink of choice for all newborn mammals from mice to humans. However, our recent findings have brought an unexpected twist: Genetic defects in the mother can lead to the secretion of inflammatory milk that is detrimental to the nursing neonates (Wan et al. 2007). Milk quality impacts the health of the offspring, as it not only regulates their postnatal development in infancy, but may also influence their propensity to develop chronic diseases in adulthood. Therefore, the understanding of the genetic and biochemical mechanisms that ensure proper milk composition will provide new insights for human physiology and diseases (Gartner et al. 2005). We hypothesize that maternal dietary defects may also trigger inflammatory milk secretion and neonatal toxicity. Because western diet (WD) consumption is associated with

[Keywords: metabolism; milk; neonatal inflammation]

${ }^{6}$ Corresponding author

E-mail yihong.wan@utsouthwestern.edu

Article is online at http://www.genesdev.org/cgi/doi/10.1101/gad.191031.112. the current epidemic of metabolic diseases such as obesity and diabetes, we investigated the consequences of maternal WD feeding on the nursing neonates in mice.

Despite the differences between human and mouse diets, a western-like rodent diet has been widely used to simulate the human WD and decipher the etiology of several metabolic diseases. Similarly, despite the differences in the composition of human and mouse milk, the major regulatory mechanisms of milk synthesis and secretion are largely conserved (Neville and Picciano 1997). Therefore, our investigations using mouse models and their associated power of genetic, dietary, and pharmacological manipulations may reveal a novel understanding of human physiology and diseases that cannot be easily obtained from epidemiological or clinical studies.

\section{Results and Discussion}

\section{Maternal WD causes neonatal toxicity and inflammation}

Wild-type female C57B6J mice were fed with either a normal chow or a western-like rodent diet containing extra fat, cholesterol, and sucrose for 2 wk before breeding with wild-type male mice and were maintained on chow or WD throughout pregnancy and lactation. Surprisingly, the pups from the WD-fed mothers (WD moms) started to lose their body hair at around postnatal day 18 (P18) (Fig. 1A); the hair loss persisted until approximately P30 and was recovered after weaning (data not shown). The pups from the WD moms also exhibited an increased body weight (Fig. 1B). H\&E staining revealed that the skin of the pups from the WD moms developed follicular cysts and a thicker fat layer compared with the skin of the pups from the chow moms (Fig. 1C). Oil Red O staining indicated that the skin of the pups from WD moms contained more lipids (Fig. 1C), which was confirmed by the significantly higher lipid/skin weight ratio (Fig. 1D). Furthermore, the skin of the pups from the WD moms exhibited precocious leukocyte infiltration (Fig. 1E) and elevated expression of inflammatory genes (Fig. 1F). Consistently, the intestines and livers of the pups from the WD moms also had increased expression of inflammatory genes (Supplemental Fig. S1). These phenotypes, such as skin inflammation, were also recovered after weaning (Fig. $1 F)$. Together, these results indicate that maternal WD feeding causes neonatal toxicity, manifested as lipid accumulation, inflammation, and alopecia.

\section{The neonatal toxicity originates from defects in lactation, rather than gestation}

To determine whether the neonatal toxicity was associated with maternal WD feeding during gestation or lactation, we performed cross-fostering experiment by switching pups between WD moms and chow moms on P2. The results showed that pups born from chow moms but nursed by WD moms developed alopecia at a penetrance comparable with pups born and nursed by WD moms. In contrast, pups born from WD moms but nursed by chow moms were largely refractory to hair loss (Fig. 2A). These results indicate that the maternal WD-mediated neonatal toxicity was caused by postnatal defects in lactation, rather than 
A

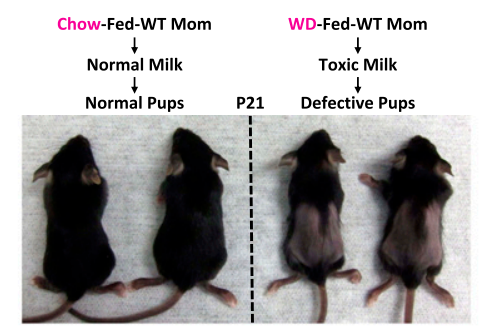

C
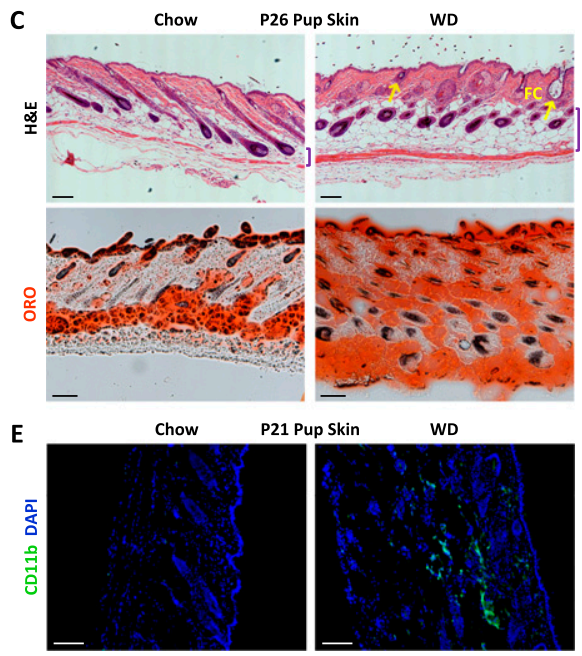

B

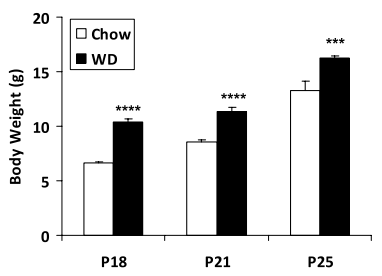

D

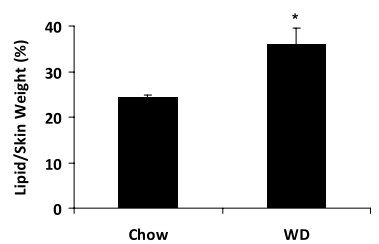

$\mathbf{F}$

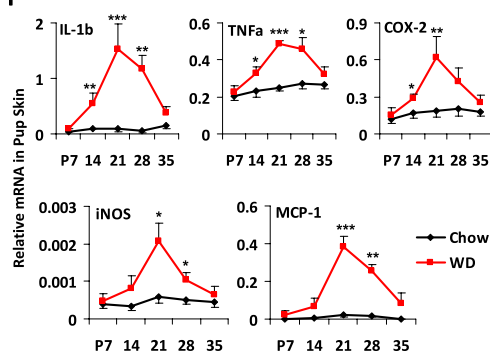

Figure 1. Maternal WD causes neonatal toxicity and inflammation. (A) Pups from WD moms developed alopecia. (B) Pups from WD moms exhibited increased body weight $(n=6)$. $(C-F)$ Defects in the skin of the pups from WD moms. $(C, t o p)$ H\&E staining shows skin hyperplasia, a thicker fat layer (indicated by bracket), and follicular cysts (FC; indicated by arrows). (Bottom) Oil Red O staining shows increased lipid accumulation. Bars, $100 \mu \mathrm{m} .(D)$ Increased lipid weight in the skin $(n=3)$. (E) Leukocyte infiltration. Skin sections were stained with FITC anti-CD11b. Bars, $25 \mu \mathrm{m}$. $(F)$ Increased inflammatory gene expression in the skin $(n=3)$.

prenatal defects in gestation. This phenotype persisted when the extra cholesterol or sucrose was individually removed from the WD (data not shown). Moreover, when the fat content of the diet was increased to $60 \%$ Kcal, the neonatal toxicity was more severe, as evidenced by lethality at P10-P20. Together, these results indicate that the excessive fat in the WD was mainly responsible. Because the WD contains not only a higher fat content, but also a higher percentage of saturated fatty acids (FAs) (Fig. 2B), we next examined lipid accumulation and FA composition in the mammary glands (MGs) and milk.

\section{Maternal WD increases long chain and saturated FAs (LcS-FAs) in milk}

$\mathrm{H} \& \mathrm{E}$ staining and Oil Red $\mathrm{O}$ staining showed that the MGs of WD moms had more and larger adipocytes as well as higher lipid content on lactation day 10 (L10) (Fig. 3A). Consistent with these results, the MG lipid/tissue weight ratio was increased by 2.4-fold (Fig. 3B), and the lipid/milk weight ratio was increased by $24 \%$ for the milk from WD moms (WD milk) compared with the milk from chow moms (chow milk) (Fig. 3C).

Triglycerides make up $98 \%$ of milk lipids, and their FAs are derived from both de novo synthesis in the lactating MG, which generates predominantly short and medium chain (C8-C12) FAs, and a maternal diet that contains

mainly long chain (C16-C20) FAs (Neville and Picciano 1997). Previous studies showed that inflammation is specifically activated by saturated FAs but not unsaturated FAs (Lee et al. 2001, 2003a,b; Shi et al. 2006; Saberi et al. 2009) and by long chain FAs (C16:0 and C18:0) but not medium/short chain FAs (C8:0-C12:0) (Hommelberg et al. 2009). Therefore, we next investigated the FA composition of milk triglycerides by shotgun lipodomic analysis using neutral loss mass spectrometry (Han and Gross 2005). The results showed that the elongation ratio (C18:0/C10:0) (Fig. 3D) was significantly increased by 1.9-fold in the WD milk compared with the chow milk, indicating a higher percentage of long chain FAs. Moreover, the saturation ratio (C18:0/C18:2) was also 2.8-fold higher in the WD milk (Fig. 3E), indicating a higher percentage of saturated FAs. These results revealed that the WD milk not only had a higher fat content, but also contained excessive LcS-FAs. To test whether the LcS-FA in the WD milk was the cause of alopecia, we supplemented pups from chow moms with either palmitic acid (C16:0) or linoleic acid (C18:2) by oral gavage, starting at P1. The results showed that excessive intake of palmitic acid, but not linoleic acid, was sufficient to cause pup hair loss (Fig. 3F).

\section{Maternal WD increases ceramides (Cer) in pups}

Saturated FAs induce the biosynthesis of Cer and glucosylceramides (GlcCer) to promote inflammation, insulin resistance, and metabolic diseases (Holland et al. 2007;

\begin{tabular}{|c|c|c|c|}
\hline $\begin{array}{c}\text { Gestation } \\
\text { Mom }\end{array}$ & $\begin{array}{l}\text { Lactation } \\
\text { Mom }\end{array}$ & $\begin{array}{l}\text { Hair Loss (\% Pup) } \\
\text { ( } n=18 \text { pups) }\end{array}$ & $\begin{array}{l}\text { Hair Loss (\% Litter) } \\
\text { ( } n=3 \text { litters) }\end{array}$ \\
\hline Chow & Chow & 0 & 0 \\
\hline WD & WD & 67 & 100 \\
\hline Chow & WD & 72 & 100 \\
\hline WD & Chow & 11 & 33 \\
\hline
\end{tabular}

\begin{tabular}{cccc} 
& & Chow & WD \\
\cline { 2 - 4 } \% by weight & Protein & 16.4 & 20.8 \\
& Carbohydrate & 48.5 & 41.9 \\
& Fat & 4.0 & 18.5 \\
\hline \% kcal from & Protein & 22.0 & 19.9 \\
& Carbohydrate & 66.0 & 40.1 \\
& Fat & 12.0 & 39.9 \\
\hline \% fatty acids & SFA & 17.6 & 48.9 \\
& MUFA & 20.6 & 38.8 \\
& PUFA & 61.8 & 12.2 \\
\hline g/kg & Sucrose & 0.0 & 152.8 \\
& Cholesterol & 0.0 & 1.7 \\
\hline & Kcal/g & 3.0 & 4.2 \\
\hline
\end{tabular}

Figure 2. Neonatal toxicity originates from defects in lactation, rather than gestation. (A) Cross-fostering of pups between chow moms and WD moms starting at P2. (B) Composition of the WD and the chow control diet. (SFA) Saturated FA, (MUFA) monounsaturated FA, (PUFA) polyunsaturated FA. 


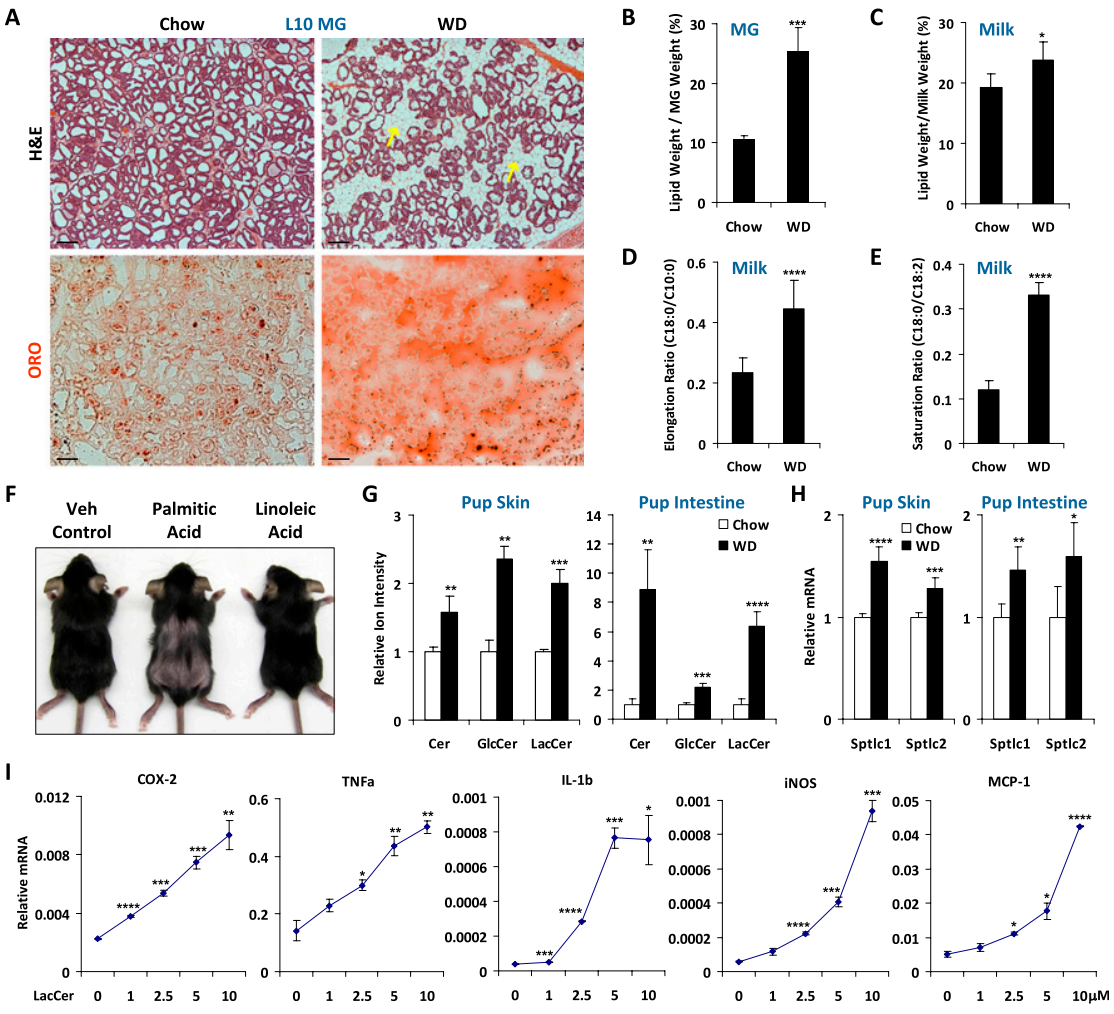

Figure 3. Maternal WD increases LcS-FAs in milk and Cer in pups. $(A)$ Increased fat in the MGs of WD moms. (Top) H\&E staining shows increased number and size of adipocytes (arrows). (Bottom) Oil Red O staining shows increased lipid accumulation. Bars, $25 \mu \mathrm{m}$. $(B)$ Quantification of the lipid weight in lactating MGs $(n=3)$. (C) Quantification of the lipid content in milk $(n=4)$. $(D, E)$ Shotgun lipodomic analyses of the FA composition in milk triglycerides by mass spectrometry. $(D)$ Ratio of long chain versus short chain FAs $(n=4)$. $(E)$ Ratio of saturated versus unsaturated FAs $(n=4)$. $(F)$ Oral supplementation of palmitic acid, but not linoleic acid, to the pups from chow moms, starting at P1, was sufficient to cause hair loss $(n=3)$. (G) Quantification of Cer, GlcCer, and LacCer in pup skin and intestines $(n=4)$. Representative results for d18:1/12:0 Cer are shown. (H) Expression of serine palmitoyl-CoA transferases in pup skin and intestines $(n=4)$. (I) LacCer-induced inflammatory genes in macrophage. RAW264.7 macrophage cell line was treated with d18:1/12:0 LacCer at the indicated concentrations for $6 \mathrm{~h}(n=3)$.

Holland and Summers 2008). To further investigate whether the LcS-FAs in the WD milk were the cause of its inflammatory property, we tested whether ingesting the WD milk increased Cer levels in the pups. Both Cer and GlcCer were significantly more abundant in the skin and intestines of the pups nursed by WD moms (Fig. 3G). Consistently, the expression of the key enzymes for Cer biosynthesis, serine palmitoyl-CoA transferase 1 (Sptlc1) and Sptlc2, was elevated in the skin and intestines (Fig. $3 \mathrm{H})$. There was also a higher level of lactosylceramide (LacCer) (Fig. 3G), which is a type of Cer incorporating lactose, a sugar rich in milk. Since LacCer has not been well characterized, we next examined whether LacCer possesses inflammatory activity in an in vitro assay using the RAW264.7 mouse macrophage cell line. The results show that the expression of a set of inflammatory genes, including COX-2, TNF $\alpha$, IL-1 $\beta$, iNOS, and MCP-1, was activated by a 6 -h LacCer treatment in a dose-dependent manner (Fig. 3I). Thus, we identified LacCer as a new class of proinflammatory lipids in the unique context of lactation. Together, these data indicate that the inflammatory activity of the WD milk resides in the excessive LcS-FAs, which elevate Cer levels in the neonates.

\section{Toll-like receptor 2/4 (TLR2/4) deletion rescues maternal WD-induced neonatal toxicity}

Previous studies have shown that both TLR2 and TLR4 mediate saturated FAinduced inflammation and insulin resistance, suggesting functional redundancy (Senn 2006; Shi et al. 2006; Poggi et al. 2007; Tsukumo et al. 2007; Caricilli et al. 2008). To further investigate whether the neonatal toxicity associated with maternal WD feeding was caused by LcS-FA-mediated inflammation, we tested whether TLR2/4 double-knockout (DKO) mice were resistant to maternal WD-induced pup hair loss. Wild-type or TLR2/4 DKO female mice were fed with WD for 2 wk before breeding to wild-type or TLR2/4 DKO male mice, respectively, and were maintained on WD throughout pregnancy and lactation. The results showed that while wild-type pups developed alopecia in all of the litters, TLR2/4 DKO pups were completely refractory to hair loss (Fig. 4A). CD11b immunofluorescence staining showed that maternal WD-mediated leukocyte infiltration in pup skin was largely abolished by TLR2/4 deletion (Fig. 4B). Gene expression analysis of a panel of proinflammatory cytokines/ chemokines/enzymes showed that maternal WD-induced inflammation in pup skin was significantly dampened by TLR2/4 deletion (Fig. 4C). Mass spectrometry analysis showed that maternal WD-induced Cer accumulation in pup skin was also attenuated by TLR $2 / 4$ deletion (Fig. 4D). Cross-fostering experiments revealed that wild-type pups still developed alopecia when fostered with TLR2/4 DKO WD moms, whereas TLR2/4 DKO pups were still resistant to hair loss when fostered with wild-type WD moms. These results indicate that TLR2/4 deletion in the pups confers resistance to the toxic milk, rather than that TLR2/4 deletion in the moms corrected the milk composition.

To determine whether the inflammatory response was mediated by TLR signaling in macrophages, we next tested mice harboring macrophage-specific deletion of the TLR adaptor protein Myd88 (Myd88 $/ \mathrm{f} / \mathrm{f} /$ ysozyme-Cre). These mice were also resistant to hair loss (Fig. 4E), suggesting that the maternal WD-induced neonatal toxicity is mainly caused by activation of the TLR2/4Myd88 signaling pathway in the macrophages.

\section{Germ-free mice remain sensitive to maternal WD-induced neonatal toxicity}

It has been shown that WD-induced metabolic disorders may involve the alteration of gut microbiota and that TLR2/4 can mediate inflammatory responses induced by not only saturated FAs, but also bacterial components (DiBaise et al. 2008; Konner and Bruning 2011). In order to determine whether gut microbiota were required for the 
A

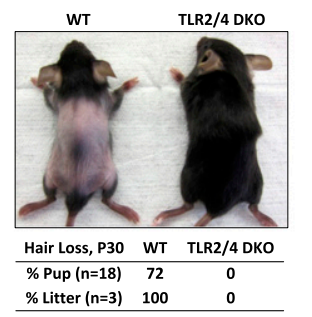
B
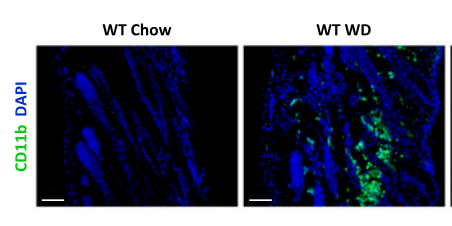

TLR2/4 DKO WD

C

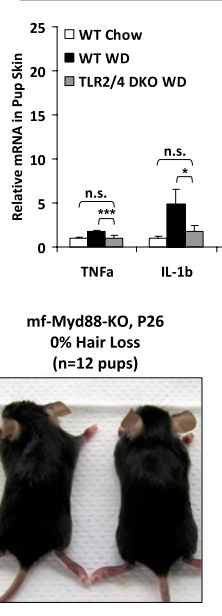
0
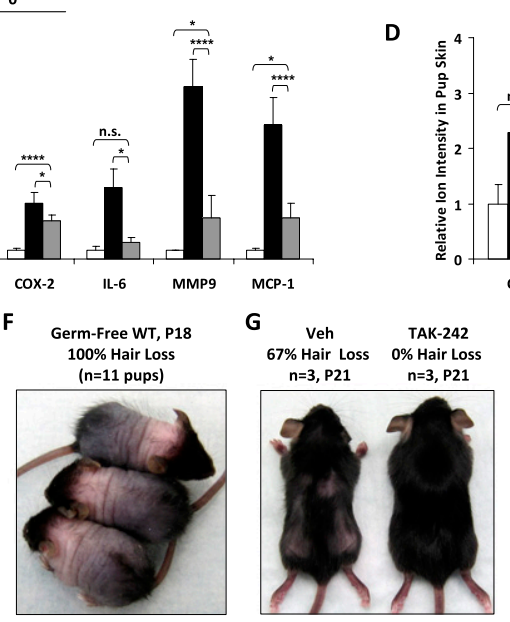

D

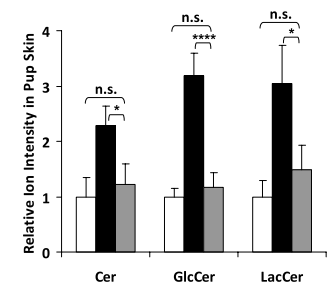

$\mathbf{H}$

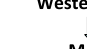

Mom Mom Toxic Milk (LCS-FA) Pup Pup TLR2/4-Myd88 $\stackrel{\searrow}{1}$ Ceramide $\Rightarrow$ Cytokines Inflammation/Toxicity

Figure 4. TLR2/4 deletion rescues maternal WD-induced neonatal toxicity. (A) TLR2/4 DKO mice were resistant to maternal WD-induced pup alopecia. (Top) A representative image of the pups. (Bottom) Penetrance of pup hair loss. $(B)$ Leukocyte infiltration in the pup skin was reduced by TLR2/4 deletion. $(C)$ Inflammatory gene expression in the pup skin was reduced by TLR2/4 deletion $(n=6)$. (D) Cer levels in the pup skin was decreased by TLR2/4 deletion $(n=3)$. (E) Macrophage $(\mathrm{mf})$ Myd88 $\mathrm{KO}$ mice were resistant to maternal WD-induced pup alopecia. $(F)$ Germ-free mice remained sensitive to maternal WD-induced pup alopecia. $(G)$ Treatment with the TLR4 inhibitor TAK-242 prevented maternal WD-induced pup alopecia. Pups from the same WD mom were orally gavaged with either TAK-242 (10mg/kg per day) or vehicle control starting at P2. $(H)$ A schematic diagram illustrating that maternal WD feeding causes the secretion of toxic milk containing excessive LcS-FAs, which activate the TLR2/4-Myd88 pathway to increase the levels of Cer as well as proinflammatory cytokines and enzymes in the nursing pups, leading to neonatal inflammation and toxicity.

maternal WD-induced neonatal toxicity, we subjected germ-free wild-type mice to the same WD feeding and breeding experimental scheme. The results show that instead of resistance, the pups nursed by WD-fed germfree moms developed a more severe phenotype, evident from an earlier onset (P15) and a higher penetrance $(100 \%$ pups and litters) of the alopecia (Fig. 4F), as well as an $80 \%$ lethality by P25. This suggests that certain intestinal bacteria may be protective to this toxicity. Together, the findings that TLR2/4 or Myd88 deletion, but not bacteria elimination, effectively rescues this phenotype provide strong in vivo and genetic evidence that the inflammatory activity in the WD milk resides mainly in the LcS-FAs.

\section{Neonatal treatment with a TLR4 inhibitor prevents maternal WD-induced toxicity}

We next investigated whether pharmacological inhibition of the TLR signaling could prevent this phenotype. Genetic rescue experiments revealed that TLR4 deletion was more effective $(0 \%$ hair loss) than TLR2 deletion (20\% hair loss); thus, we tested the effect of a synthetic
TLR4 inhibitor, TAK-242 (Li et al. 2006; Sha et al. 2007). Pups from the same WD mom were divided into two groups and orally gavaged with either TAK-242 (10mg/kg per day) or vehicle control starting at P2. While $67 \%$ of the pups in the vehicle-gavaged control group developed alopecia, none of the pups in the TAK-242-treated group exhibited hair loss (Fig. 4G). This result indicates that maternal WDinduced neonatal toxicity can be effectively treated by TLR4 inhibitors.

In summary, here we show that maternal WD feeding causes the secretion of inflammatory milk that contains excessive LcS-FAs, which activate the TLR2/4-Myd88 signaling pathway to elevate the levels of Cer as well as proinflammatory cytokines and enzymes, consequently triggering neonatal toxicity (Fig. $4 \mathrm{H})$. Therefore, conceptually, our findings unravel the WD-induced lactation lipotoxicity and inflammatory milk as a novel aspect of the metabolic syndrome at the maternal offspring interface. Understanding how maternal defects such as WD consumption lead to toxic milk secretion may have implications in the etiology and treatment of neonatal inflammatory disorders.

Mother's milk represents a unique metabolic entity that exerts direct consequences on the developing newborns, whose digestive and immune systems are still immature. Indeed, our findings reveal that neonates are very sensitive to the alterations of milk lipid composition and that excessive LcS-FAs in the milk causes toxicity and inflammation, manifested as a visually identifiable transient alopecia phenotype. Importantly, we gained key mechanistic understanding of this abnormality by demonstrating that TLR $2 / 4$ DKO mice, but not germ-free mice, are resistant to this alopecia. Therefore, technically, our study highlights the milk-neonate axis as a novel in vivo experimental system to identify new genetic or biochemical factors that regulate lipid metabolism and inflammation.

\section{Materials and methods}

\section{Mice and diet}

TLR2/4 DKO mice on C57B6 background have been described (Hoshino et al. 1999; Takeuchi et al. 1999). Wild-type C57B6, lysozyme-cre (Clausen et al. 1999), and Myd88 flox (Hou et al. 2008) mice were from Jackson Laboratory. Mice were fed ad libitum with irradiated chow or WD that had been tested under various culture conditions to be free of bacteria and fungi (Harlan Laboratories). The WD was whole-grain-based, as was the chow control diet, in order to support pregnancy and lactation and contained a composition of extra fat, cholesterol, and sucrose similar to that of the typical synthetic WD. Germ-free C57B6 mice were maintained in plastic gnotobiotic isolators as described (Cash et al. 2006). For MG, milk, and pup analyses, 8- to 10 -wk-old female mice were prefed with WD or chow control for 2 wk before breeding and were maintained on WD or chow throughout pregnancy and lactation. The litter sizes were normalized to six pups. Milk was collected as described (Schwertfeger et al. 2003; Wan et al. 2007). For FA supplementation, pups from the same chow mom were orally gavaged with palmitic acid or linoleic acid at $100 \mathrm{mg} / \mathrm{kg}$ per day or vehicle control. For TLR4 inhibitor treatment, pups from the same 
WD mom were orally gavaged with TAK-242 (CLI-095, Invivogen) at $10 \mathrm{mg} / \mathrm{kg}$ per day or vehicle control. All protocols for mouse experiments were approved by the Institutional Animal Care and Use Committee of University of Texas Southwestern Medical Center.

\section{Histology and staining}

For histological analysis, the tissues were immediately fixed in 10\% PBSbuffered formalin overnight, then processed, paraffin-embedded, sectioned, and H\&E-stained according to standard protocols. For Oil Red O staining, the tissue samples were immediately frozen in OCT compound in a dry ice/ethanol bath and stored at $-80^{\circ} \mathrm{C}$ until sectioning. The frozen sections were fixed in formalin for $10 \mathrm{~min}$, dipped in $60 \%$ isopropanol, and then stained in Oil Red O working solution $(60 \% 3 \mathrm{mg} / \mathrm{mL}$ Oil Red O in isopropanol) for $15 \mathrm{~min}$. For immunofluorescence staining, frozen OCT sections were fixed in acetone for $10 \mathrm{~min}$ and stained with FITCconjugated antibodies specific for $\mathrm{CD} 11 \mathrm{~b} / \mathrm{Mac}-1 \alpha$ (BD Biosciences). The sections were washed twice and mounted with medium containing DAPI (Vector Laboratories).

\section{Gene expression analyses}

Tissue samples were snap-frozen in liquid nitrogen and stored at $-80^{\circ} \mathrm{C}$. RAW264.7 macrophages were treated with d18:1/12:0 LacCer (Avanti Polar Lipids) or vehicle control for $6 \mathrm{~h}$. RNA was extracted using Trizol (Invitrogen), treated with RNase-free DNase I, reverse-transcribed into cDNA using an ABI High-Capacity cDNA RT kit, and analyzed using realtime quantitative PCR (SYBR Green) in triplicate. All RNA expression was normalized by L19.

\section{Lipid analysis by mass spectrometry}

Organic soluble metabolites were extracted from tissue or milk samples with a 2:1:1 $\mathrm{CHCl}_{3} / \mathrm{MeOH} / \mathrm{H}_{2} \mathrm{O}$ solution and homogenization as described (Wan et al. 2007). Samples were stored at $-80^{\circ} \mathrm{C}$ and dissolved in $\mathrm{CHCl}_{3}$ prior to analysis. Cer were quantified by LC-MS/MS in positive ionization multiple reaction monitoring (MRM) mode as described (Sullards and Merrill 2001). Shotgun lipidomic analyses were performed using an Agilent Triple Quadrupole mass spectrometer under neutral loss mode as described (Han and Gross 2005). A C17:0 triglyceride standard (Sigma) was added to each sample at $100 \mathrm{nmol} / \mathrm{mg}$ prior to lipid extraction and was used to normalize the results.

\section{Statistical analyses}

All statistical analyses were performed with Student's $t$-test and are represented as mean \pm standard deviation $\left(\right.$ s.d.); $\left.\left(^{*}\right) P<0.05 ;{ }^{*}{ }^{\star *}\right) P<0.01$; $\left(^{(\star \star}\right) P<0.005 ;\left(^{\star \star \star \star}\right) P<0.001$; (n.s.) not significant $(P>0.05)$.

\section{Acknowledgments}

We thank Chandrashekhar Pasare at University of Texas Southwestern Medical Center for TLR2/4 DKO mice, David Mangelsdorf and Joel Elmquist at University of Texas Southwestern Medical Center for discussion, University of Texas Southwestern Medical Center Molecular Pathology Core for histology, and Sunia Trauger at the Small Molecule Mass Spectrometry Facility of Harvard FAS Center for Systems Biology for assistance with lipid analysis. Y.W. is a Virginia Murchison Linthicum Scholar in Medical Research and a recipient of the Basil O'Connor Scholar Award. A.S. is a recipient of the Burroughs Wellcome Career Award in Biosciences, a Searle Scholars Award, and an Alfred P. Sloan Award. L.V.H. is an investigator of the Howard Hughes Medical Institute. This work was supported by the March of Dimes (\#5-FY10-1 to Y.W.), the Welch Foundation (I-1751 to Y.W.), CPRIT (RP100841 to Y.W.), the NIH (R01 DK089113 to Y.W., and R01 DK070855 to L.V.H.), and University of Texas Southwestern Medical Center Endowed Scholar Startup Fund (to Y.W.).

\section{References}

Caricilli AM, Nascimento PH, Pauli JR, Tsukumo DM, Velloso LA, Carvalheira JB, Saad MJ. 2008. Inhibition of toll-like receptor 2 expression improves insulin sensitivity and signaling in muscle and white adipose tissue of mice fed a high-fat diet. J Endocrinol 199: 399406.

Cash HL, Whitham CV, Behrendt CL, Hooper LV. 2006. Symbiotic bacteria direct expression of an intestinal bactericidal lectin. Science 313: $1126-1130$.

Clausen BE, Burkhardt C, Reith W, Renkawitz R, Forster I. 1999. Conditional gene targeting in macrophages and granulocytes using LysMcre mice. Transgenic Res 8: 265-277.

DiBaise JK, Zhang H, Crowell MD, Krajmalnik-Brown R, Decker GA, Rittmann BE. 2008. Gut microbiota and its possible relationship with obesity. Mayo Clin Proc 83: 460-469.

Gartner LM, Morton J, Lawrence RA, Naylor AJ, O'Hare D, Schanler RJ, Eidelman AI. 2005. Breastfeeding and the use of human milk. Pediatrics 115: 496-506.

Han X, Gross RW. 2005. Shotgun lipidomics: Electrospray ionization mass spectrometric analysis and quantitation of cellular lipidomes directly from crude extracts of biological samples. Mass Spectrom Rev 24: $367-412$.

Holland WL, Summers SA. 2008. Sphingolipids, insulin resistance, and metabolic disease: New insights from in vivo manipulation of sphingolipid metabolism. Endocr Rev 29: 381-402.

Holland WL, Brozinick JT, Wang LP, Hawkins ED, Sargent KM, Liu Y, Narra K, Hoehn KL, Knotts TA, Siesky A, et al. 2007. Inhibition of ceramide synthesis ameliorates glucocorticoid-, saturated-fat-, and obesity-induced insulin resistance. Cell Metab 5: 167-179.

Hommelberg PP, Plat J, Langen RC, Schols AM, Mensink RP. 2009. Fatty acid-induced NF- $\mathrm{BB}$ activation and insulin resistance in skeletal muscle are chain length dependent. Am I Physiol Endocrinol Metab 296: E114-E120. doi: 10.1152/ajpendo.00436.2007.

Hoshino K, Takeuchi O, Kawai T, Sanjo H, Ogawa T, Takeda Y, Takeda K, Akira S. 1999. Cutting edge: Toll-like receptor 4 (TLR4)-deficient mice are hyporesponsive to lipopolysaccharide: Evidence for TLR4 as the Lps gene product. J Immunol 162: 3749-3752.

Hou B, Reizis B, DeFranco AL. 2008. Toll-like receptors activate innate and adaptive immunity by using dendritic cell-intrinsic and -extrinsic mechanisms. Immunity 29: 272-282.

Konner AC, Bruning JC. 2011. Toll-like receptors: Linking inflammation to metabolism. Trends Endocrinol Metab 22: 16-23.

Lee JY, Sohn KH, Rhee SH, Hwang D. 2001. Saturated fatty acids, but not unsaturated fatty acids, induce the expression of cyclooxygenase-2 mediated through Toll-like receptor 4. I Biol Chem 276: 16683-16689.

Lee JY, Plakidas A, Lee WH, Heikkinen A, Chanmugam P, Bray G, Hwang DH. 2003a. Differential modulation of Toll-like receptors by fatty acids: Preferential inhibition by $\mathrm{n}-3$ polyunsaturated fatty acids. J Lipid Res 44: 479-486.

Lee JY, Ye J, Gao Z, Youn HS, Lee WH, Zhao L, Sizemore N, Hwang DH. 2003b. Reciprocal modulation of Toll-like receptor-4 signaling pathways involving MyD88 and phosphatidylinositol 3-kinase/AKT by saturated and polyunsaturated fatty acids. I Biol Chem 278: 3704137051.

Li M, Matsunaga N, Hazeki K, Nakamura K, Takashima K, Seya T, Hazeki O, Kitazaki T, Iizawa Y. 2006. A novel cyclohexene derivative, ethyl (6R)-6-[N-(2-chloro-4-fluorophenyl)sulfamoyl]cyclohex-1-ene1-carboxylate (TAK-242), selectively inhibits toll-like receptor 4-mediated cytokine production through suppression of intracellular signaling. Mol Pharmacol 69: 1288-1295.

Neville MC, Picciano MF. 1997. Regulation of milk lipid secretion and composition. Annu Rev Nutr 17: 159-183.

Poggi M, Bastelica D, Gual P, Iglesias MA, Gremeaux T, Knauf C, Peiretti F, Verdier M, Juhan-Vague I, Tanti JF, et al. 2007. C3H/HeJ mice carrying a toll-like receptor 4 mutation are protected against the development of insulin resistance in white adipose tissue in response to a high-fat diet. Diabetologia 50: 1267-1276.

Saberi M, Woods NB, de Luca C, Schenk S, Lu JC, Bandyopadhyay G, Verma IM, Olefsky JM. 2009. Hematopoietic cell-specific deletion of toll-like receptor 4 ameliorates hepatic and adipose tissue insulin resistance in high-fat-fed mice. Cell Metab 10: 419-429.

Schwertfeger KL, McManaman JL, Palmer CA, Neville MC, Anderson SM. 2003. Expression of constitutively activated Akt in the mammary gland leads to excess lipid synthesis during pregnancy and lactation. J Lipid Res 44: 1100-1112. 
Senn JJ. 2006. Toll-like receptor-2 is essential for the development of palmitate-induced insulin resistance in myotubes. J Biol Chem 281: $26865-26875$.

Sha T, Sunamoto M, Kitazaki T, Sato J, Ii M, Iizawa Y. 2007. Therapeutic effects of TAK-242, a novel selective Toll-like receptor 4 signal transduction inhibitor, in mouse endotoxin shock model. Eur J Pharmacol 571: 231-239.

Shi H, Kokoeva MV, Inouye K, Tzameli I, Yin H, Flier JS. 2006. TLR4 links innate immunity and fatty acid-induced insulin resistance. J Clin Invest 116: 3015-3025.

Sullards MC, Merrill AH Jr. 2001. Analysis of sphingosine 1-phosphate, ceramides, and other bioactive sphingolipids by high-performance liquid chromatography-tandem mass spectrometry. SCi STKE 2001: pl1. doi: 10.1126/stke.2001.67.pll.

Takeuchi O, Hoshino K, Kawai T, Sanjo H, Takada H, Ogawa T, Takeda K, Akira S. 1999. Differential roles of TLR2 and TLR4 in recognition of Gram-negative and Gram-positive bacterial cell wall components. Immunity 11: 443-451.

Tsukumo DM, Carvalho-Filho MA, Carvalheira JB, Prada PO, Hirabara SM, Schenka AA, Araujo EP, Vassallo J, Curi R, Velloso LA, et al. 2007. Loss-of-function mutation in Toll-like receptor 4 prevents dietinduced obesity and insulin resistance. Diabetes 56: 1986-1998.

Wan Y, Saghatelian A, Chong LW, Zhang CL, Cravatt BF, Evans RM. 2007. Maternal PPAR $\gamma$ protects nursing neonates by suppressing the production of inflammatory milk. Genes Dev 21: 1895-1908. 


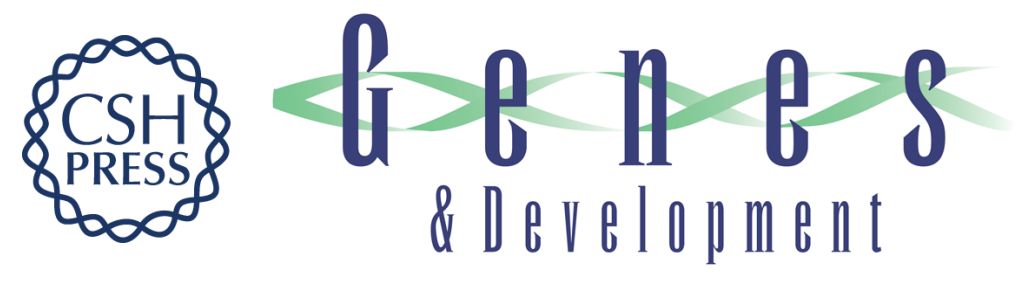

\section{Maternal western diet causes inflammatory milk and TLR2/4-dependent neonatal toxicity}

Yang Du, Marie Yang, Syann Lee, et al.

Genes Dev. 2012, 26:

Access the most recent version at doi:10.1101/gad.191031.112

Supplemental http://genesdev.cshlp.org/content/suppl/2012/06/19/26.12.1306.DC1
Material

References This article cites 27 articles, 12 of which can be accessed free at:

http://genesdev.cshlp.org/content/26/12/1306.full.html\#ref-list-1

License

Email Alerting Receive free email alerts when new articles cite this article - sign up in the box at the top

Service right corner of the article or click here.

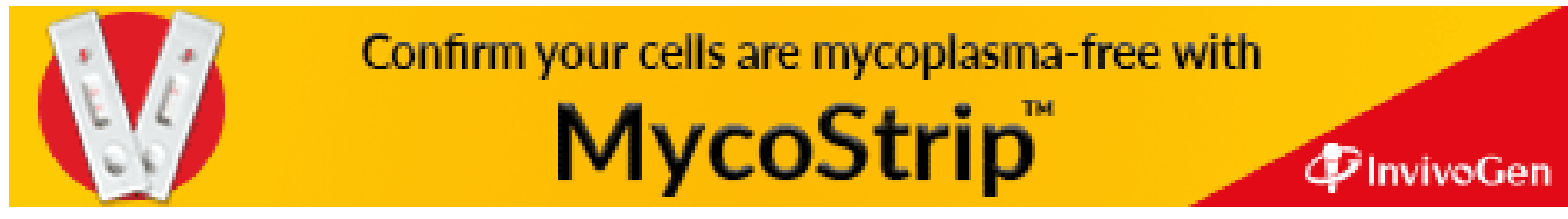

